\title{
Concurso CORVI para viviendas en Valparaiso: comentario sobre el proyecto ganador, el Conjunto Habitacional "Villa Italia"
}

[1967] Housing in Valparaiso CORVI competition: coments on the winning entry "Villa Italia"

\section{Autor}

Alfonso Raposo M. [1967]

\section{Filiación}

Centro de Estudios Arquitectónicos, Urbanísticos y del Paisaje de la Universidad Central

\section{Resumen}

La reedición de este artículo busca recoger la línea de diseño urbano residencial que se desarrollaba en la Facultad de Arquitectura y Urbanismo en esa época.

\section{Palabras Clave}

Diseño Urbano, Vivienda Urbana, Concurso de Arquitectura, CORVI, Conjunto Habitacional

\section{Abstract}

This competition entry presents the urban housing design taught at that time (1967) in FAU.

\section{Keywords}

Urban Design, Housing, Architecture Competition, CORVI, Urban Dwelling.

\section{Sumario}

0.- Introducción

1. El significado y uso de la información de las bases del Concurso.

2. Propósitos y objetivos de diseño arquitectónico según los autores del proyecto "Villa Italia"

3. Análisis de las viviendas

4. Comentario sobre el fallo del jurado

\section{Introducción}

El día 23 de Marzo del año 1966 se conoció el resultado del Concurso de Anteproyectos organizado por la Corporación de la Vivienda, correspondiente a un conjunto de viviendas para la "Villa Italia" en Valparaíso. El proyecto ganador correspondió a los arquitectos Eduardo San Martín y Pedro Pascal.

Por el interés que presenta su solución, se da a continuación algunos antecedentes sobre este proyecto. 


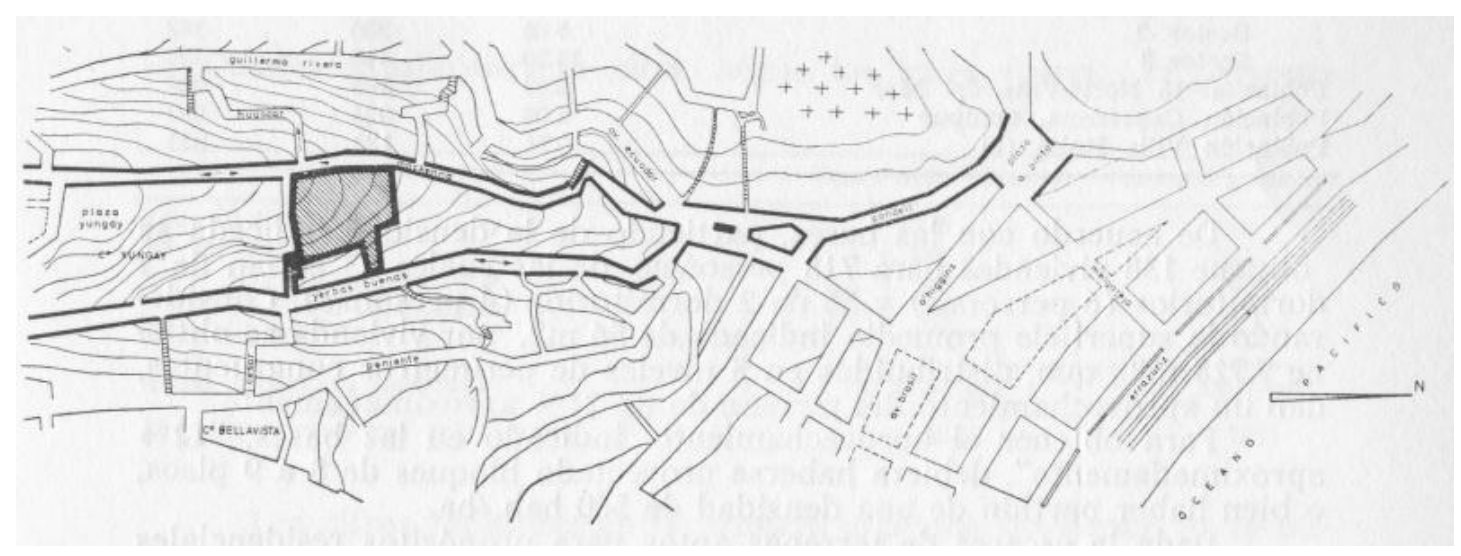

Figura 1.- Croquis de ubicación del proyecto "Villa Italia".

\section{El significado y uso de la información de las bases del Concurso.}

Se da una densidad bruta máxima de 970 habitantes por hectárea, que en sí es una cifra bastante alta para conjuntos residenciales ${ }^{1}$. Sin embargo, considerando la superficie del terreno de $7.400 \mathrm{~m} 2$., el empleo de esta densidad no significaría crear una zona extensa con tal concentración de pobladores sino solamente una pequeña área como podría serlo un edificio o grupo de edificios, cuya gravitación en cuanto a demanda de servicios de sus pobladores puede suponerse absorbida por la oferta existente en la estructura urbana circundante.

A título de comparación cabe señalar las densidades obtenidas en otros conjuntos residenciales.

\begin{tabular}{|l|r|r|r|}
\hline \multicolumn{3}{|c|}{$\begin{array}{l}\text { CUADRO DE COMPARACIÓN DE DENSIDADES EN DIFERENTES CONJUNTOS } \\
\text { REDSIDENCIALES, INCLUIDO "VILLA ITALIA". }\end{array}$} \\
\hline Conjuntos residenciales & $\begin{array}{l}\text { Superficie } \\
\text { en há. }\end{array}$ & $\begin{array}{l}\text { No viviendas } \\
\text { Densidad } \\
\text { Hab./há. }\end{array}$ \\
\hline Población Ignacio Zenteno, Valparaíso & 1,92 & 185 & 560 \\
\hline Edificio Brasil, Valparaíso & 6,42 & 200 & 194 \\
\hline Población Quebrada Verde, Valparaíso: & & & 149 \\
\hline Sector 1 & 16,00 & 538 & 125 \\
\hline Sector 2 & 9,05 & 330 & 820 \\
\hline Sector 3 & 14,30 & 448 & 697 \\
\hline Población 15 Norte-Viña del Mar & 2,19 & 300 & 935 \\
\hline Población Capredena, Quilpué & 2,06 & 234 & 132 \\
\hline Población Villa Italia & 0,74 & & \\
\hline
\end{tabular}

1 El "Plan de Nuevas Obras" 1966 de la CORVI establece una densidad máxima de 780 hab/ha. Cuando se construye en 5 pisos, como patrón habitacional regional.

${ }^{2}$ Cifras obtenidas por el proyecto ganador. 
De acuerdo con las bases, partiendo de la densidad indicada se obtiene 138 viviendas para 718 personas, de las cuales 83 serían de 3 dormitorios ( 6 personas) y 55 de 2 dormitorios ( 4 personas). Considerando la superficie promedio indicada de $56 \mathrm{~m}^{2}$ por vivienda se obtiene $7.728 \mathrm{~m}^{2}$, que, distribuidos en 5 niveles de perímetros congruentes, dan un aprovechamiento del terreno de un $21 \%$ aproximadamente.

Para obtener el aprovechamiento indicado en las bases, "12\% aproximadamente", debiera haberse proyectado bloques de 8 a 9 pisos, o bien haber partido de una densidad de 560 hab/ha.

Dada la escasez de terrenos aptos para propósitos residenciales en Valparaíso, es comprensible la necesidad planteada en las bases de emplear altas densidades y edificios colectivos, aún cuando no ha sido suficientemente solventada la vocación de la familia de ingresos medios para la forma de vida en departamentos $y$, por el contrario, pueden enumerarse algunas evidencias a favor de una inaptitud.

La alternativa de proporcionar viviendas en predios individuales de acuerdo con la estructura normativa actual comienza a ser posible, en este caso, al reducir la densidad a 310 habitantes por hectárea, lográndose con ello 40 viviendas aproximadamente.

La posibilidad de aumentar la densidad de población sin recurrir a bloques de departamentos, manteniendo la noción de viviendas unifamiliares, puede lograrse (considerando las viviendas nominalmente como departamentos para los efectos legales normativos, eludiendo con ello el requerimiento de disponer predios de una superficie mínima de 160 metros), mediante un proyecto complejo, de diferentes tipos de volúmenes dispuestos en diversas combinaciones de adyacencia y superposición para conformar pequeños patios y terrazas individuales ${ }^{3}$.

Para una hipotética solución de este carácter, resuelta en dos niveles, en que se utilizara un $30 \%$ del terreno en edificación, se tendría 80 viviendas y una densidad de $560 \mathrm{~h} / \mathrm{ha}$, quedando sin edificar $12 \mathrm{~m}^{2}$., por persona aproximadamente.

La dificultad de este tipo de soluciones radica principalmente en la cuantía del metraje indicado para las viviendas, que obliga a un diseño compacto, disminuyendo las posibilidades de juego volumétrico.

El "Plan de Nuevas Obras 1966" de la CORVI fija como patrón habitacional una superficie de 50 a $56 \mathrm{~m}^{2}$ para las "viviendas medias".

\footnotetext{
${ }^{3}$ Algunos ejemplos extranjeros de estas soluciones: Grupo de Viviendas "Atrium" en Chicago; grupo "Habitat 67", en Montreal; conjunto de viviendas en Torguay, Revista "L'Architecture d'aujourd'hui", No 103 y 120.
} 


\begin{tabular}{|l|c|c|}
\hline \multicolumn{2}{|c|}{$\begin{array}{c}\text { CUADRO COMPARATIVO DE CIFRAS EN LAS BASES DEL CONCURSO Y EN EL } \\
\text { PROYECTO "VILLA ITALIA". }\end{array}$} \\
\hline & Bases del Concurso & Proyecto ganador \\
\hline Densidad hab/ha & 979 & 935 \\
\hline No de viviendas & 138 & 132 \\
\hline De 3 dormitorios & 83 & 83 \\
\hline De 2 dormitorios & 55 & 702 \\
\hline No de habitantes & 718 & $7.897 \mathrm{~m}^{2}$ \\
\hline Total superficie edificada & $7.730 \mathrm{~m}^{2}$ & $19 \%$ \\
\hline$\%$ aprovechamiento del terreno & $12 \%$ & \\
\hline
\end{tabular}

Las cifras indicadas en la primera columna provienen de la utilización de la densidad máxima 970 h/ha. Como puede apreciarse, las cifras obtenidas por el proyecto ganador se ciñen con muy cercana correspondencia a esta determinación.

Otras cifras obtenidas en el proyecto que nos ocupa son las siguientes:

Superficie $\mathrm{m}^{2}$ de estacionamiento (42 vehículos)

Superficie $\mathrm{m}^{2}$ de circulación del peatón

\section{Propósitos y objetivos de diseño arquitectónico según los autores del proyecto "Villa Italia""}

PARTIDO GENERAL. El proyecto pretende:

Asimilarse a la topografía espacial de Valparaíso

Los edificios y espacios de Valparaíso tienen unas dimensiones, proporciones y escala que esta proposición pretende mantener para asimilarse a la topografía arquitectónica de la ciudad. Esto se consigue mediante la construcción de edificios pequeños adaptados a los diferentes niveles del terreno, que generan un conjunto de volúmenes y espacios similares a los existentes en los cerros de la ciudad.

Mantener los valores espaciales del terreno

La topografía, las vistas, las circulaciones naturales del terreno y la ciudad a sus pies, le dan un sentido espacial al terreno que el proyecto quiso relevar. Se pretende destacar estos valores estableciendo un sistema de agrupación de edificios y terrazas miradores que se abren hacia la ciudad y el mar.

\footnotetext{
${ }^{4}$ Texto redactado por los arquitectos San Martín y Pascal, para el presente artículo, [1967].
} 
Adaptarse a los cotas del terreno

El proyecto se adapta a las cotas del terreno mediante:

-Bloques pequeños que por las dimensiones de su planta pueden adaptarse mejor en terrenos con desnivel.

-Utilización de las plataformas existentes en el terreno.

-Ubicación de los accesos de los edificios en diferentes niveles para aprovechar las diferencias de cotas.

Organizar un sistema de agrupación de edificios

Los edificios están agrupados en dos sistemas que forman una trama que se desprende hacia la ciudad, la que a su vez facilita la creación de diferentes tipos de espacios y su construcción por etapas de acuerdo a las exigencias establecidas en las bases del concurso. Esos sistemas están formados por edificios con una escalera por articulación lo que permite reconocerlos individualmente, aprovechar las vistas del terreno y ubicarse en diferentes niveles de altura.

Mantener la vegetación existente en el terreno:

Los edificios se han proyectado de modo de respetar la vegetación existente en el terreno.

Con respecto a los departamentos el proyecto pretende:

Emplear dos tipos de departamentos modulados

Se diseñaron dos tipos de departamentos, uno de 6 camas y otros de 4 camas, los que se adaptan a cualquiera orientación y permiten la reducción de los costos de construcción al repetir unidades similares. La planificación de los departamentos está modulada de modo de permitir el empleo al máximo de elementos estandarizados tales como moldajes prefabricados modulados para los hormigones, tabiques interiores, puertas y ventanas, unidades sanitarias, etc.

Reducción de las circulaciones comunes

La forma de agrupaciones de los edificios y el diseño de los departamentos reduce al máximo las circulaciones comunes, las que se diseñaron al aire libre para disminuir su costo.

\section{Empleo de una estructura económica}

Se diseñó una estructura económica utilizando sólo los elementos estructurales necesarios, lo que permite el máximo de utilización de elementos no resistentes y de igual libertad para la construcción del resto del edificio. 
REVISTA DE

URBANISMO

ISSN 0717-5051

http://revistaurbanismo.uchile.cl

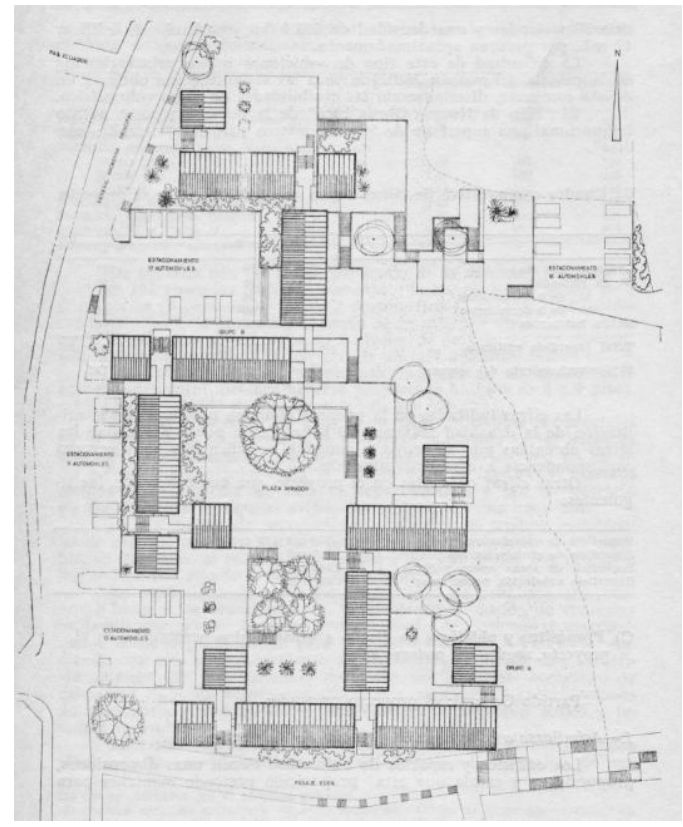

Figura 2.- Plano del conjunto "Villa Italia".

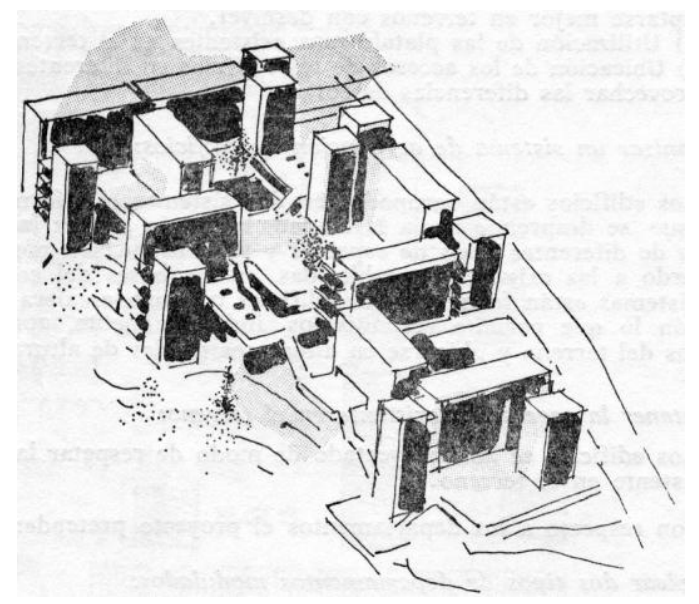

Figura 3.- El conjunto "Villa Italia". 


\section{Análisis de las viviendas}

Se dan a continuación los cuadros de superficie de los departamentos según el siguiente desglose: 1.- La superficie habitable diurna que incluye el estar-comedor. 2.- La superficie habitable nocturna que incluye los dormitorios. 3.- La superficie de servicio que incluye la cocina, el lavadero, la sala de baño y los closets, y 4.- La superficie de circulación. La suma de estos cuatros rubros da la superficie útil.

Se dan en la primera columna los $\mathrm{m}^{2}$, en la segunda el porcentaje que esos $\mathrm{m}^{2}$ representan de la superficie útil total, y en la tercera columna, una cifra que representa el \% de habitabilidad ${ }^{5}$.

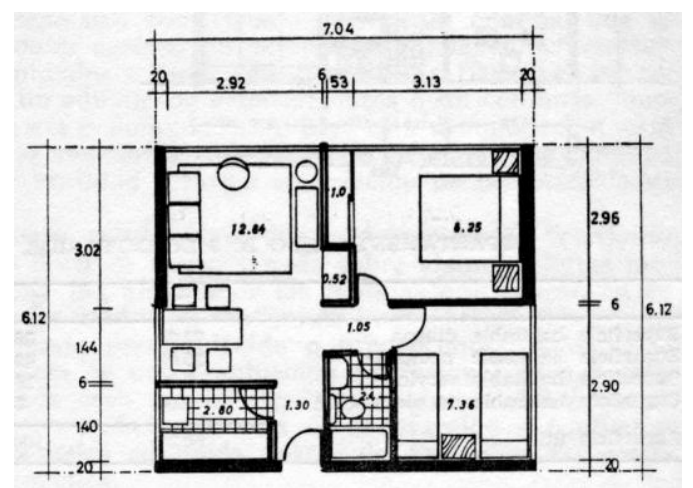

Figura 4.-Departamento Tipo A: 2 dormitorios, 4 camas. "Villa Italia".

\begin{tabular}{|l|r|r|r|}
\hline \multicolumn{3}{|c|}{ DEPARTAMENTO TIPO A: 2 DORMITORIOS, 4 CAMAS. } \\
\hline \multicolumn{2}{|c|}{$\mathrm{m}^{2}$} & \multicolumn{1}{c|}{$\%$ sup. } & \multicolumn{1}{c|}{$\begin{array}{c}\text { \% } \\
\text { habitabilidad }\end{array}$} \\
\hline Superficie habitable diurna & 12,27 & 33,3 & 54,7 \\
\hline Superficie habitable nocturna & 15,61 & 42,4 & 42,2 \\
\hline Superficie de servicio & 6,66 & 18,0 & 21,7 \\
\hline Superficie de circulación & 2,37 & 6,3 & 12,9 \\
\hline Superficie útil & 36,89 & 100,0 & \\
\hline
\end{tabular}

\footnotetext{
${ }^{5}$ Porcentaje determinado según un método de evaluación del uso del espacio en la vivienda elaborada en
} IVUPLAN y basado en un informe del Director del Instituto Nacional de la Vivienda de Bélgica, M. Lebegge. 
http://revistaurbanismo.uchile.cl

A título de comparación se dan los siguientes porcentajes de habitabilidad obtenidos en otras viviendas CORVI de programa similar:

Tipo 608: 125,8

Tipo 622: 123,6

Tipo 651: 126,5

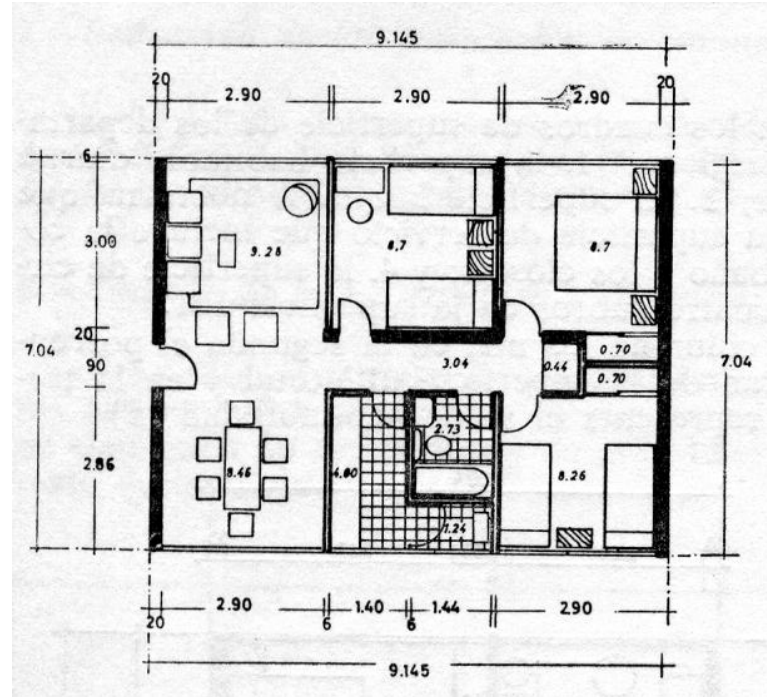

Figura 5.-Departamento tipo B: 3 dormitorios, 6 camas. "Villa Italia".

\begin{tabular}{|l|r|r|r|}
\hline \multicolumn{3}{|c|}{ DEPARTAMENTO TIPO B: 3 DORMITORIOS, 6 CAMAS } \\
\hline & \multicolumn{1}{|c|}{$\mathrm{m}^{2}$} & \multicolumn{1}{c|}{$\%$ sup. } & \multicolumn{1}{c|}{$\begin{array}{c}\text { \% } \\
\text { habitabilidad }\end{array}$} \\
\hline Superficie habitable diurna & 20,60 & 35,0 & 42,70 \\
\hline Superficie habitable nocturna & 25,69 & 43,3 & 43,10 \\
\hline Superficie habitable servicio & 9,86 & 16,6 & 16,28 \\
\hline Superficie habitable de circulación & 3,04 & 5,1 & 7,60 \\
\hline Superficie útil & 59,27 & 100,0 & 109,68 \\
\hline
\end{tabular}

Los porcentajes de habitabilidad obtenidos en viviendas análogas a éstas son los siguientes:

Tipo 401 - A: 119,3

Tipo 400 - A: 116,1

Tipo $500-A: 116,3$ 


\section{$4 . \quad$ Comentario sobre el fallo del jurado}

El juicio que solventa la elección de un proyecto ganador es naturalmente el juicio sobre el óptimo estado funcional de las proposiciones formuladas por los concursantes, es decir, el juicio sobre la solución más adecuada para los propósitos del concurso dentro de las determinantes expuestas en las bases del mismo.

El propósito del concurso, aun cuando no especificado, se encontraba implícito en su denominación Conjunto Habitacional "Villa Italia" - Valparaíso. Se trataba pues de hacer aquello que se sobreentiende es un conjunto habitacional, a saber: dentro de la estructura física espontánea de la ciudad, ínsulas planificadas y de materialización homogénea en el tiempo y el espacio, que se apoyan parcial o totalmente en el medio circundante; dentro del marco arquitectónico, agregados de viviendas que conforman un medio formalmente deliberado, unitario y reconocible, que satisface exigencias del habitar cotidiano de las familias que desarrollan allí su vida dentro del ambiente doméstico y fuera de él.

Pero estos elementos utilizados aquí para describir a un conjunto habitacional son válidos tanto para describir dos viviendas pareadas, o un edificio de departamentos, como para las proposiciones presentadas por los concursantes para Villa Italia.

Hay, por consiguiente, dos posibilidades: o la denominación conjunto habitacional tiene una consistencia puramente nominal que se da en general a cualquier agrupación de viviendas, independientemente de la cantidad de unidades y modo de conformación física y, en tal caso, tanto tres casas, un edificio de departamentos o un conjunto "operación sitio" aceptan esa denominación, o bien esta denominación está reservada para señalar aquellas agrupaciones de viviendas que cumplen ciertos requisitos de cantidad y forma en relación de las necesidades de las familias.

En el primer caso, resulta claro que la denominación "conjunto habitacional", por no decir ni suponer nada sobre algunas ciertas maneras de satisfacer las necesidades de las familias que vivirán en él, ni sobre algunos ciertos modos de configurar la estructura física para tal fin, no alude a nada preestablecido o preconceptualizado, y se trataría, en consecuencia, de una nominación operacional cuya consistencia es dada en cada caso por el arquitecto a través del proceso: conocimiento de las necesidades, modos de satisfacción y configuración de la estructura física adecuada dentro del marco de las determinaciones circunstanciales.

En la segunda posibilidad, por el contrario, estaría implícito en la denominación el contar con elementos ya preestablecidos y preconceptualizados, lo que supone haber previamente explorado el conocimiento respecto a la relación entre: necesidades, modo de satisfacción y marco físico.

En cualquier caso, sea cual fuere el verdadero significado de la denominación conjunto habitacional, lo cierto es que de todos modos es necesario remitirse al conocimiento de las necesidades y modo de satisfacción. 
En otras palabras, un conjunto habitacional será más conjunto y más habitacional en la medida que satisfaga, dentro del marco arquitectónico y urbanístico, su propósito, esto es, las exigencias del habitar de las familias que vivan en él.

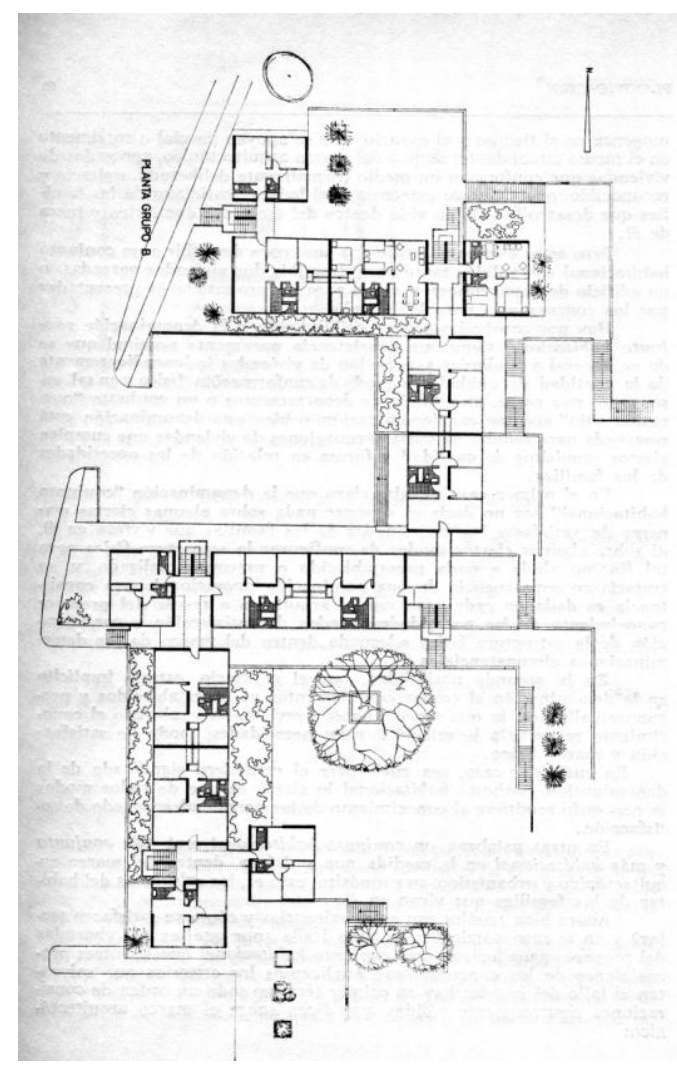

Figura 6.- Planta Grupo B. "Villa Italia".

Ahora bien, ¿cuáles son estas exigencias y cómo se satisfacen mejor? Y en el caso particular de Villa Italia, ¿por qué las 132 viviendas del proyecto ganador son más conjunto habitacional que las otras proposiciones de los concursantes? Analicemos los criterios que solventan el fallo del jurado.

Hay en primer término todo un orden de consideraciones rigurosamente válidas que dicen sobre el marco arquitectónico:

- El aprovechamiento de las características topográficas del terreno.

- El juego diverso y ordenado de volúmenes.

- La nítida unidad formal.

- Los valores ambientales logrados.

- La simplicidad y adaptabilidad de las viviendas tipo, su modulación y sencillez estructural.

- La doble orientación y facilidades de ventilación. 
- La vista directa al panorama predominante.

- La cualidad de reflejar la forma de vida del cerro de Valparaíso o, quizás más propiamente, la cualidad de reflejar la forma de vida en cuanto a movilidad y percepción, puesto que la cualidad de reflejar en una estructura física la forma de vida característica del cerro de Valparaíso en su consistencia sociológica, parece, dentro de lo que es instrumentalidad actual del arquitecto, una afirmación aventurada.

- La explotación positiva de las diferencias de nivel.

- La buena situación de los miradores.

- La proporción del marco arquitectónico.

En segundo término, respecto a las cualidades habitacionales en su significación social se dice:

- Las circulaciones de peatones organizadas hacia espacios exteriores compacto que favorecen la convivencia de grupos.

Y de los criterios generales del acto oficial del Fallo habría que señalar:

- El empleo activo de los espacios exteriores generados por la agrupación de volúmenes, condición importante para un conjunto de alta densidad.

- El fraccionamiento en numerosos bloques versus la exaltación de grandes volúmenes y la consecuente expresión social masificada.

Dos parecen ser las exigencias habitacionales principales subyacentes en los conceptos del jurado: la necesidad de convivencia general y la necesidad de privacidad individual de las familias.

Pero hay diversas formas de convivencia y privacidad que van desde la simple no agresión física, visual y auditiva basada en el respeto a las personas, hasta la convivencia activa que supone la existencia de organizaciones más o menos formales en que la privacidad consiste tanto en la libertad de participar o no en ellas, como también en no ser interferido por sus actividades.

Privacidad y convivencia son nociones válidas en un orden conceptual, pero su validez en un plano de realidad depende de su ponderación en el modo de vida.

Generalmente el arquitecto tiene un acceso directo al conocimiento del modo de vida (sistematizado en el complejo - necesidades - actividades - disponibilidades), del sujeto para quien proyecta. Pero en el caso de los conjunto habitacionales el sujeto es anónimo y el eventual conocimiento que permite deducir diseño se realiza indirectamente a través de la información que entrega el organismo patrocinador de la acción habitacional, en el caso particular de un concurso, debemos suponer, a través de las bases. 


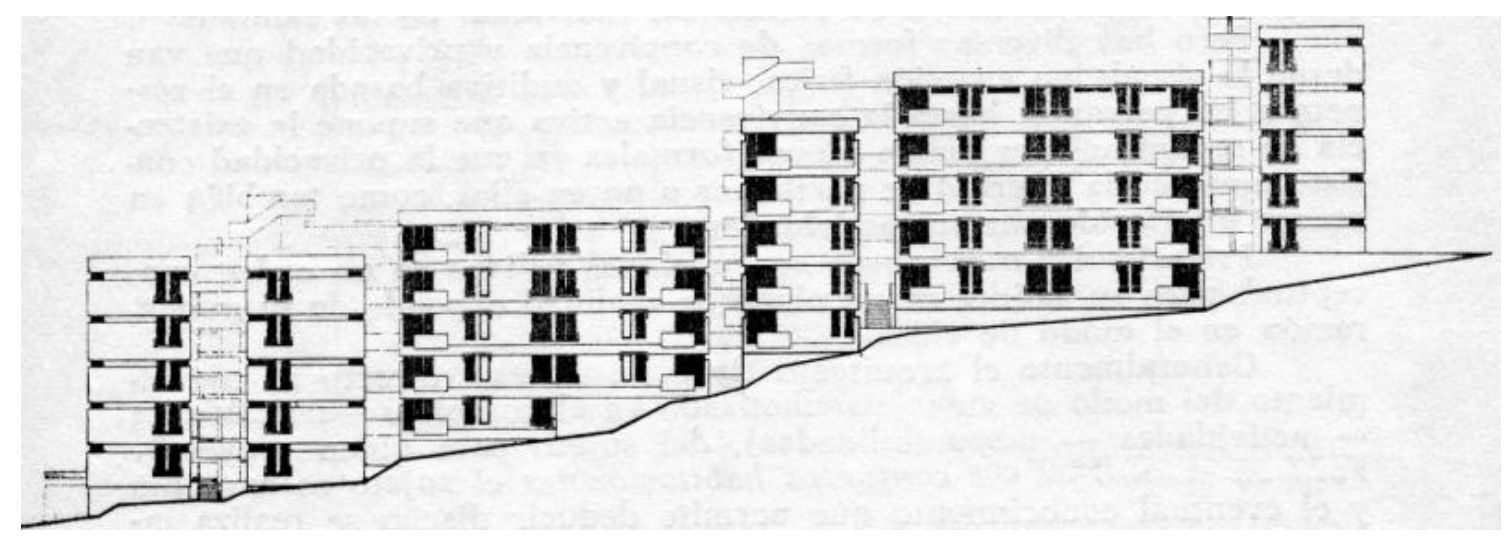

Figura 7.-Aspecto parcial del alzado poniente de "Villa Italia".

El problema podría consistir en que no se conozca realmente la forma de vida (y las transformaciones que pueda experimentar) en su dimensión social, de los futuros ocupantes del conjunto habitacional, en tal caso cualquier proposición física se estaría haciendo con un margen bastante grande de incertidumbre.

La planificación de conjuntos habitacionales puede contener en sí, acciones homogeneizantes que impiden cada vez más atender a las peculiaridades individuales, pero sólo es el desconocimiento de estas peculiaridades lo que hace que parte de las formas poblacionales realizadas impongan, con consecuencias a veces desafortunadas, formas de vida no homogéneas a la naturaleza de las personas.

Creemos que el conjunto habitacional Villa Italia será un caso afortunado, que en efecto, la configuración de "espacios activos" y el encauce de los moradores a tales espacios favorece la convivencia y que "el fraccionamiento en numerosos bloques" promete valores de privacidad.

Un estudio de este conjunto en el futuro permitirá verificar su éxito.

Fte.: RAPOSO MOYANO, Alfonso. 1967. "Comentario sobre el conjunto habitacional 'Villa Italia' ". En: Revista de Planificación. Vivienda. Ciudad. Región. Instituto de la Vivienda, Urbanismo y Planeación N4, Facultad de Arquitectura y Urbanismo de la Universidad de Chile, enero de 1967, pp. 84-96. 